\title{
Gravitational equilibrium moisture profiles in swelling soils
}

\author{
Jagath C. Ekanayake \\ Manaaki Whenua-Landcare Research New Zealand Ltd., Christchurch, New Zealand \\ David J. Painter \\ Department of Natural Resources Engineering, Lincoln University, Canterbury, New Zealand
}

\begin{abstract}
Extension of the work of Philip (1969a, 1972) has shown that the effect of overburden pressure on volume change of swelling soils produces equilibrium moisture profiles entirely different from those predicted without considering the overburden pressure effects. Volume change of swelling soils accompanied by moisture changes also affects the overburden pressure. The total volume change under an overburden pressure occurs in three different shrinkage phases from saturation to oven dry. These volume change characteristics are usually described on the shrinkage surface, a surface drawn as void ratio $e$ (volume of voids/volume of solids) versus moisture ratio $\Theta$ (volume of water/ volume of solids) for different overburden pressures. A relationship for the moisture gradient is developed by assuming that the overburden potential, a component of the total potential of soil water, is a function of moisture ratio only across a small soil element of a long swelling soil column which consists of large numbers of finite soil elements. The moisture gradient has complex behavior based on the properties of the three shrinkage phases on the shrinkage surface. Distribution of equilibrium moisture paths is then explained by evaluating the moisture gradients at points on an idealized shrinkage surface. It is shown that the soil at great depths could either be saturated or unsaturated at equilibrium. (These depths could be over $250 \mathrm{~m}$ for some sodium montmorillonite clays).
\end{abstract}

\section{Introduction}

The theory of the hydrology of swelling soil is different from that of rigid soil due to volume change with moisture content under overburden pressure [Philip, 1969a, b, c, 1972]. In rigid soil, moisture content increases toward the water table where the soil becomes saturated. Below the water table, the saturated moisture content remains unchanged with depth at equilibrium. The equilibrium moisture content in swelling soils, however, could even decrease with depth below the water table due to volume change under the overburden soil pressure. Such moisture profiles have been observed in the field by Talsma [1974].

Introducing an additional potential component to the total potential of soil water in rigid soil, Philip [1969a] described three types of equilibrium moisture profiles in swelling soils. The new potential component, which was similar to that suggested by Coleman and Croney [see Philip, 1969a], was called the "overburden potential" $\Omega(\Theta)$, defined by $(d e / d \Theta) P$, where $P$ is the overburden pressure and $d e / d \Theta$ is the slope of the shrinkage curve $e(\Theta)$ for zero overburden pressure. Using the characteristics of volume change determined by earlier experimental data, Philip [1969a] suggested that all three types of moisture profiles reach a unique moisture ratio $\Theta_{p}$ (picnotatic point), which is always unsaturated at great depths and where the apparent wet relative density of the soil reaches its maximum. The overburden pressure was assumed to have a negligible effect on the volume change, and such assumptions created uncertainties about the existence of unsaturated zones Copyright 1995 by the American Geophysical Union.

Paper number 95WR01652.

0043-1397/95/95WR-01652\$05.00 below shallow water tables [Youngs and Towner, 1970]. Although the load factor was corrected to include the effect of overburden pressure on the volume change as given by (1) [Philip, 1970; Groenevelt and Bolt, 1972], the equilibrium moisture distribution in swelling soils has not been corrected accordingly.

The load factor $\alpha_{n}$ is

$$
\alpha(\Theta, P)_{n}=P_{n}^{-1} \int_{0}^{P_{n}}\left[\frac{\partial e}{\partial \Theta}\right]_{P, \Theta, T} d P
$$

for the $n^{\text {th }}(i=n)$ soil element under a constant overburden pressure $P_{n} ; e$ is the void ratio, $T$ is the absolute temperature, and $\Theta$ is the moisture ratio. Here " $i$ "' indexes soil elements of finite thickness beginning at the surface $(i=0)$ and increases with depth (Figure 1).

The work presented in this paper examines the behavior of equilibrium moisture profiles in swelling soil, considering the volume change properties affected by both moisture ratio and overburden pressure [Ekanayake, 1991]. Such equilibrium moisture profiles are described using an idealized shrinkage diagram based on the properties of the previously reported experimental data of volume change (see below). Final settlements of civil engineering structures could be predicted using the relationship $e(\Theta, P)$, if the equilibrium moisture profile is evaluated using the shrinkage curves obtained for a sufficient range of overburden pressures.

\section{Theory}

\subsection{General Properties of Swelling Soils} and the Shrinkage Diagram

From earlier theoretical and experimental results the following characteristics of volume change of swelling soil under 

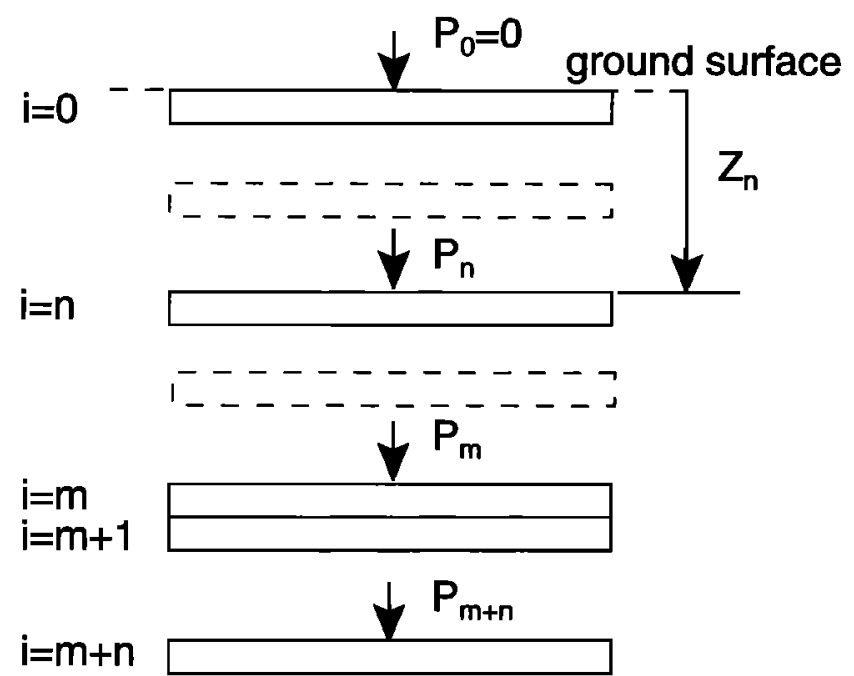

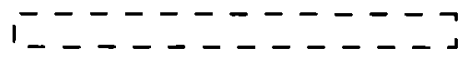

Figure 1. Idealized swelling soil profile. Here " $i$ " indexes soil segments of finite thickness beginning at the surface $(i=$ 0 ) and increases with depth to minimum void ratio at $i=m$.

overburden pressure are considered to be valid and applicable for swelling soils which exhibit one-dimensional and reversible volume changes [Tempany, 1917; Haines, 1923; Lauritzen and Stewart, 1941; Holmes, 1955; Warkentin and Bozozuk, 1961; Fox, 1964; Philip and Smiles, 1969; Philip, 1969a, 1971, 1972; Groenevelt and Bolt, 1972; Sposito, 1973; Talsma, 1977; Giraldez et al., 1983; Giraldez and Sposito, 1983].

Volume change of a swelling soil under an overburden pressure takes place in three shrinkage phases from saturation to oven dry at a moisture ratio equal to $\Theta_{0}$. Volume reduction is no longer possible for moisture ratios below $\Theta_{0}$. Such volume change behavior can be described on a single shrinkage curve $e_{n}(\Theta)$ (say for the $n^{\text {th }}$ soil element, $(i=n)$ ), obtained for a constant overburden pressure $P_{n}$ as normal, residual, and zero shrinkage phases.

The normal shrinkage phase is associated with the saturated condition, where void ratio is equal to the moisture ratio and change of moisture and void volumes are equal. Therefore $e=$ $\Theta$ and $(\partial e / \partial \Theta)_{P n}=1$ for $\Theta \geq \Theta_{a n}$ where $\Theta_{a n}$ is the air entry point under the overburden pressure $P_{n}$.

The residual shrinkage phase occurs during further drying, after air begins entering the system at the air entry point $\Theta_{a n}$ and before the shrinkage limit $\Theta_{z n}$. In the residual shrinkage phase, void ratio is larger than the moisture ratio, and the change of void volume is less than the change of moisture volume. Therefore $e>\Theta$ and $(\partial e / \partial \Theta)_{P n}<1$ for $\Theta_{a n}>\Theta>\Theta_{z n}$.

The zero shrinkage phase occurs after the shrinkage limit $\Theta_{z n}$, where the final void ratio under the overburden pressure $P_{n}$ remains unchanged at $e_{0 n}$ with further removal of moisture and therefore $(\partial e / \partial \Theta)_{P n}=0$ for $\Theta_{z n}>\Theta>\Theta_{0}$.

These characteristics are valid for overburden pressures, $P_{i}=P_{0}, P_{1}, \cdots, P_{n}, P_{n+1}, \cdots, P_{m}, P_{m+1}, \cdots,(i=0$, $1, \cdots, n, n+1, \cdots, m, m+1, \cdots, P=P_{\max }$ for $\left.i=m\right)$.

Consolidation studies of Wyoming bentonite with $90-95 \%$ montmorillonite show that the final irreducible void ratio $\left(e_{0 m}\right)$ under saturated conditions could reach beyond 44 bars of pressure [Mesri and Olson, 1971]. This is approximately equal to a soil depth of $250 \mathrm{~m}$, with no surface loads.
The shrinkage diagram $e(\Theta, P)$ (Figure 2) shows a collection of shrinkage curves $e_{t}(\Theta)$ for a series of soil elements (Figure 1) beginning from an unloaded soil surface as $i=$ $0, \cdots, n-1, n, n+1, \cdots, m, \cdots, m+1, \cdots,(m$ is max, see below).

The void ratio of swelling soils decreases with overburden pressure [Chang and Warkentin, 1968; Groenevelt and Bolt, 1972; Yong and Warkentin, 1975; Talsma, 1977; Giraldez et al., 1983; Giraldez and Sposito, 1983]. During moisture removal the void ratio at any given moisture ratio reaches a minimum at a large overburden pressure, say at $\boldsymbol{P}_{\max },(i=m)$, and this void ratio is no longer reducible with further increase of overburden pressure; therefore the shrinkage curves, $e_{m}(\Theta), e_{m+1}(\Theta)$, $e_{m+2}(\Theta), \cdots$, take a unique shape for $P \geq P_{\max },(i \geq m)$.

Normal shrinkage phases of all the shrinkage curves, $e_{t}(\Theta)$ for $i=0,1, \cdots, n, n+1, \cdots, m, m+1, \cdots$, create the load surface on the $e(\Theta, P)$ diagram. On the load surface, soil is saturated and $e=\Theta, d e / d P=d \Theta / d P$ for $P>0$ and $(\partial e / \partial \Theta)_{P n}=1$ [Groenevelt and Bolt, 1972]. Shrinkage curves have a unique shape for $P \geq P_{\max }$ and $(\partial e / \partial P)_{\Theta}=0$, for $P \geq P_{\text {max }}$.

Below the air entry points, $\left(\Theta<\Theta_{a n}\right)$ for all the soil elements $(i=0,1, \cdots, n, n+1, \cdots, m, m+1, \cdots)$, the $e(\Theta, P)$ surface creates the residual surface where $(\partial e / \partial P)_{\Theta}<0$ for $0<P<P_{\max }$ and $(\partial e / \partial P)_{\Theta}=0$, for $P \geq P_{\max }$, because shrinkage curves have a unique shape for $P \geq P_{\max }$.

The load factor $\alpha_{n}$, given by (1), is related to the average of $(\partial e / \partial \Theta)_{\Theta}$ for overburden pressures $P_{i}, i=0,1, \cdots, n$ for $\Theta>\Theta_{0}$. On the load surface, $\alpha_{n}=(\partial e / \partial \Theta)_{P n}=1$ for $\Theta>\Theta_{a 0}$ for $P \geq 0$. Therefore $\alpha_{n}<(\partial e / \partial \Theta)_{P n, \Theta}$ for $\Theta_{a 0}>\Theta>\Theta_{0}$. And because of the compressibility of swelling soil, $(\partial e / \partial \Theta)_{\Theta}$ and $\alpha_{n}$ increase with overburden pressure for $\Theta_{a 0}>\Theta>\Theta_{0}$. $\Theta_{a 0}$ is the air entry value for zero overburden pressure.

Since the void ratio is a nondecreasing function of the moisture ratio, $\left(\partial^{2} e / \partial \Theta^{2}\right)_{P n}>0$ in the residual phase [Philip, 1969a], and therefore $(\partial \alpha / \partial \Theta)_{P n}>0$ for $\Theta_{a 0}>\Theta>\Theta_{z n}$. And also because $(\partial e / \partial \Theta)_{P_{n}}=1$ in the normal shrinkage phase and $(\partial e / \partial \Theta)_{P n}=0$ in the zero shrinkage phase, $\left(\partial^{2} e / \partial \Theta^{2}\right)_{P n}=0$ and $\left(\partial \alpha_{n} / \partial \Theta\right)_{P n}=0$ within the zero and normal shrinkage phases.

The total potential of soil water $\Phi_{T}$ across the $n^{\text {th }}$ soil element $(i=n)$, with a finite thickness under a constant overburden pressure $P_{n}$ (head equivalent), varies according to

$\Phi_{T}=\Psi(\Theta)-\left(Z_{n}+z\right)+\alpha_{n}(\Theta)\left[P_{n}+\int_{0}^{z} \gamma_{n}(\Theta) d z\right]$

where $\left(Z_{n}+z\right)$ is the gravitational potential (head equivalent, vertical ordinate taken positive downward from the top soil surface to the top of the $n^{\text {th }}$ soil element and $z$ is measured from the top surface of the $n^{\text {th }}(i=n)$, soil element). Here $\alpha_{n}$ is the load factor given by (1), and $\gamma_{n}(\Theta)$ is the apparent wet relative density (wet bulk density of soil/density of water). Both $\alpha_{n}$ and $\gamma_{n}$ are assumed to be unique functions of $\Theta$ across the finite soil element under the constant overburden pressure $P_{n}$. $\Psi(\Theta)$ is the matric potential of the unloaded soil (head equivalent) and is a unique function of the moisture ratio [Philip, 1969a; Towner, 1981]. (Note that $\alpha_{n}$ and $\gamma_{n}$ are determined for overburden pressure $P_{n}$ ).

The moisture gradient across the $n^{\text {th }}$ soil element at equilibrium ( $\Phi_{T}$ constant) can be obtained from (2) as 


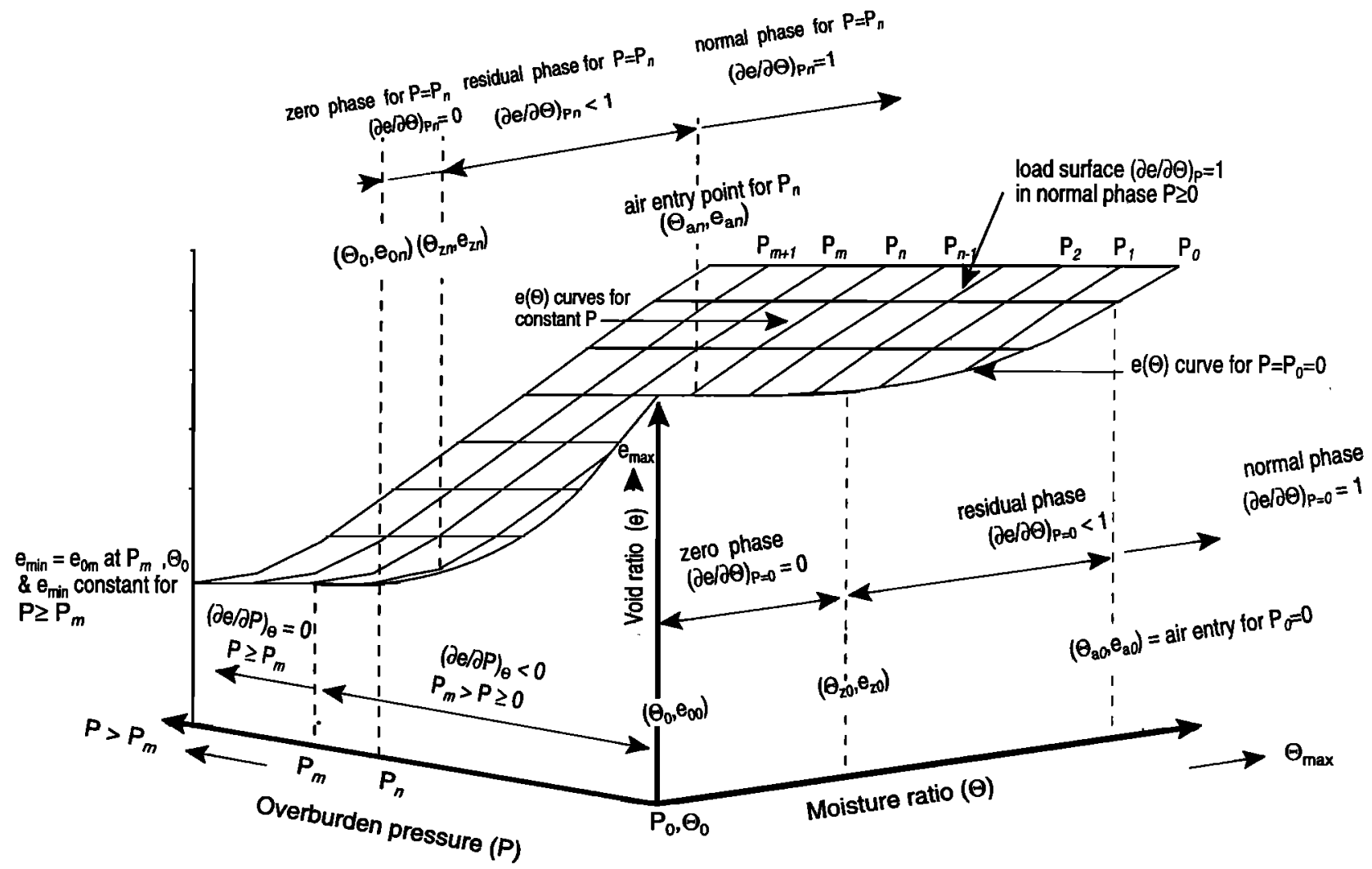

Figure 2. Idealized shrinkage diagram $e(\Theta, P) . \Theta_{a n}, e_{a n}$ is the moisture ratio and void ratio at air entry point for $P_{n} ; \Theta_{z n}, e_{z n}$ is the shrinkage limit for $P_{n} ;$ and $e_{\min }$ is the irreducible void ratio at $\Theta_{0}$ for $P_{\text {max }}$.

$$
\begin{aligned}
\frac{d \Theta}{d z} & =\frac{\left[1-\alpha_{n}(\theta) \gamma_{n}(\theta)\right]}{\left[\left(\frac{d \Psi}{d \Theta}\right)+\frac{d \alpha_{n}(\theta)}{d \Theta}\left(P_{n}+\int_{Z_{n}}^{Z_{n+1}} \gamma_{n}(\Theta) d z\right)\right]} \\
& =\frac{1}{M_{n}(\Theta)}
\end{aligned}
$$

where $Z_{n+1}-Z_{n}=\Delta Z$ is the thickness of the small soil element.

At large depths the term $\int_{Z_{n}+1}^{Z_{n+1}} \gamma(\Theta) d z$ is a small quantity compared to large overburden pressures and may be ignored in calculating $M_{n}(\Theta)$. Approximating $\left(P_{n+1}-P_{n}\right)$ by $\int_{Z_{n}}^{Z_{n+1}} \gamma(\Theta) d z$ and using $d P / d \Theta=\gamma_{n} d z / d \Theta$ across the $n^{\text {th }}$ soil element, equilibrium moisture profiles are simply traced on the $e=0$ plane by evaluating $d z / d \Theta=M_{n}(\Theta)$ across each soil element, beginning from the surface soil element $i=0$ at equilibrium with a known surface moisture ratio $\Theta_{P 0}$, and surface overburden pressure $P_{0}$ and continuing $i=1, \cdots, n, n+1, \cdots$, $m, m+1, \cdots$ toward large depths where $P>P_{m}$. To achieve this, the behavior of $M_{n}(\Theta)$ must be well understood. The functions $\gamma_{n}(\Theta), \alpha_{n}(\Theta), d \Psi / d \Theta$, and $\alpha_{n} \gamma_{n}(\Theta)$ will now be examined separately and then together.

The apparent wet relative density $\gamma_{n}(\Theta)$, which is given by (4), is determined for a swelling soil under overburden pressure $P_{n}$. It is always greater than unity for $\Theta \geq \Theta_{0}$ but approaches unity as $\Theta \rightarrow \Theta_{\max }$, where $\Theta_{\max }$ is a large quantity greater than the saturated moisture ratio without overburden pressure $-\Theta_{\max } \ggg \Theta_{a 0}$. Figure 3 shows the idealized functions: $e_{n}(\Theta), \gamma_{n}(\Theta)$, and $\alpha_{n} \gamma_{n}(\Theta)$ for soil elements $i=0, n$, and $m$.

$$
\gamma_{n}(\Theta)=\frac{\left(\Theta+\gamma_{s}\right)}{\left(1+e_{n}(\Theta)\right)}
$$

where $\gamma_{s}$ is the relative density of solids.

Here $\gamma_{n}(\Theta)$ has only one maximum given by $\gamma_{d n}\left(\Theta_{d n}\right)=$ $1 /(\partial e / \partial \Theta)_{P n}$, at $\Theta_{d n}$ for $\Theta_{z n}<\Theta<\Theta_{a n}$ [Philip, 1969a]. Because the void ratio decreases with overburden pressure, $\gamma_{n}(\Theta)$ increases with overburden pressure at any given moisture ratio. Similarly to the behavior of the $e_{n}(\Theta)$ curve, the $\gamma_{n}(\Theta)$ curve approaches the $\gamma_{m}(\Theta)$ curve as $P$ approaches $P_{m}$. Thereafter the general characteristics of $\gamma_{m}(\Theta)$ remain unchanged with $P$ (Figures $3 \mathrm{a}$ and $3 \mathrm{~b}$ ).

The load factor $\alpha_{n}(\Theta)$ given by (1) is always less than unity for $\Theta<\Theta_{a 0}$ but $\alpha_{n}(\Theta) \rightarrow 1$ as $\Theta \rightarrow \Theta_{a 0}$. It is also clear from (1) and the general properties of volume change described above that $\alpha_{n}(\Theta)=0$ for $\Theta_{z n}>\Theta>\Theta_{0}$ (zero shrinkage phase) and $\alpha_{n}(\Theta)<1$ for $\Theta_{z n}<\Theta<\Theta_{a n}$ (residual shrinkage phase). Therefore, similarly to the behavior of the $\gamma_{n}(\Theta)$ and $e_{n}(\Theta)$ curves, $\alpha_{n}(\Theta)$ also approaches the $\alpha_{m}(\Theta)$ curve as $P \rightarrow P_{m}$ where its shape remains unchanged for $P \geq P_{m}$.

From (3) at a certain moisture ratio, say $\Theta_{I n}$, $\gamma_{n}\left(\Theta_{I n}\right) \alpha_{n}\left(\Theta_{I n}\right)=1$ and $M_{n}\left(\Theta_{I n}\right) \rightarrow \pm \infty$. It has been shown that $\alpha_{n}<(\partial e / \partial \Theta)_{P n, \Theta}$ for $\Theta_{a 0}>\Theta>\Theta_{0}$ and both functions increase from 0 at $\Theta_{0}$ to 1 as $\Theta \rightarrow \Theta_{a 0}$. Therefore the condition $(\partial e / \partial \Theta)_{P n} \Theta_{n}=1$ at $\Theta_{d n}$ must be satisfied before the condition $\alpha_{n} \gamma_{n}=1$ is satisfied at $\Theta_{I n}$ in $\Theta<\Theta_{a 0}$. And also as $\Theta \rightarrow \Theta_{\max }$, the function $\alpha_{n} \gamma_{n}(\Theta)$ approaches unity as both $\gamma_{n}(\Theta)$ and $\alpha_{n}(\Theta)$ approach 1 at large moisture ratios. Therefore $\Theta_{I n}$ lies between the air entry point $\Theta_{a 0}$ and maximum apparent wet relative density point $\Theta_{d n}$ (Figure 3c).

For all swelling soils we take $d \Psi / d \Theta>0$ for $\Theta_{\max }>\Theta \geq \Theta_{0}$ 

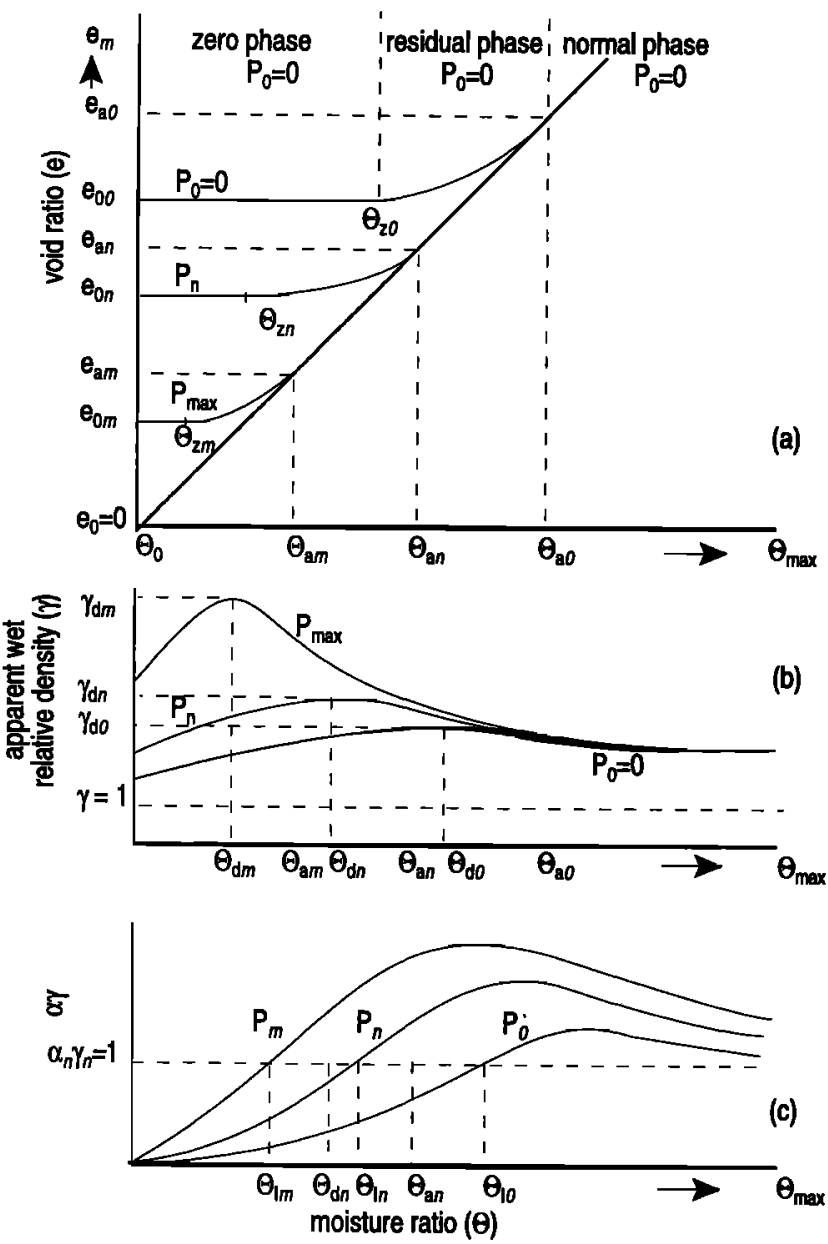

Figure 3. Idealized functions of (a) $e(\Theta)$, (b) $\gamma(\Theta)$, and (c) $\alpha \gamma(\Theta)$ for overburden pressures, $P_{0}=0, P_{n}$, and $P_{\max }$. Normal, residual, and zero shrinkage phases are shown for $P_{0}=0$ in Figure 3a.

[Philip, 1969a; Towner, 1981]. Equation (3) describes the behavior of the moisture gradient $d z / d \Theta=M_{n}(\Theta) . M_{n}(\Theta)>0$ for $\Theta<\Theta_{l n}$ and approaches $+\infty$ as $\Theta \rightarrow \Theta_{I n} . M_{n}(\Theta)<0$ for $\Theta>\Theta_{I n}$ and $M_{n}(\Theta)$ approaches $-\infty$ as $\Theta$ approaches $\Theta_{I n}$ from $\Theta_{a n}$; also note that $\left(\partial M_{n}(\Theta) / \partial \Theta\right)_{P n}>0$ for $\Theta>\Theta_{I n}$.

\subsection{The Functions $e\left(\Theta_{a}, \mathbf{P}_{a}\right)$ and $e\left(\Theta_{,}, P_{I}\right)$}

All shrinkage curves for soil elements $i=0,1,2, \cdots$, $n, \cdots, m, \cdots, m+1, \cdots$ have air entry points at the moisture ratios $\Theta_{a 0}, \Theta_{a 1}, \Theta_{a 2}, \cdots, \Theta_{a n}, \cdots, \Theta_{a m}, \Theta_{a m+1}$ and also $M_{n}(\Theta)=d z / d \Theta \approx d P / d \Theta \rightarrow \pm \infty$ at moisture ratios $\Theta_{I 0}, \Theta_{I 1}, I 2, \cdots, \Theta_{I n}, \cdots, \Theta_{I m}, \cdots, \Theta_{I m+1}$. All these points form two curves $e\left(\Theta_{a}, P_{a}\right)$ and $e\left(\Theta_{I}, P_{I}\right)$ on the shrinkage surface. Because the moisture ratios $\Theta_{a n}$ and $\Theta_{/ n}$ decrease with the overburden pressure until $\boldsymbol{P}=\boldsymbol{P}_{\max }$, these two curves are concave to the $P$ axis, and since the shrinkage curves have a unique shape for $P \geq P_{\max }$, they become parallel to the $P$ axis for $P \geq P_{\max }$. These two curves can be drawn on the $e=0$ plane, as $P\left(\Theta_{a}\right)$ and $P\left(\Theta_{I}\right)$ (Figures $4 \mathrm{a}$, 5a, and 6a). It must be noted that according to the properties of $M_{n}(\Theta)$ described in the previous paragraph, $d \Theta / d P\left[=(\partial \Theta / \partial P)_{e=0}\right]>0$ for $P(\Theta)<P\left(\Theta_{I}\right), d \Theta / d P<0$ for $P(\Theta)>P\left(\Theta_{I}\right)$, and on $P\left(\Theta_{l}\right)$, $d \Theta / d P \approx d \Theta / d z=0$.

\subsection{The Location of $\boldsymbol{\Theta}_{I n}$}

It has already been shown that $\Theta_{a 0}>\Theta_{I n}>\Theta_{d n}$. It is also important to know the exact location of the point $\Theta_{I n}$ to trace the equilibrium moisture profiles because $\Theta_{I n}$ could be either on the load surface $\left(\Theta_{I n}>\Theta_{a n}\right)$ or on the residual surface $\left(\Theta_{I n}<\Theta_{a n}\right.$ ) (note that $\left.\Theta_{d n}<\Theta_{a n}<\Theta_{a 0}\right)$. It is certain that $\Theta_{I n}$ (at which $d \Theta / d P=0$ ) cannot lie on the load surface because on an ideal load surface, soil is saturated $(e=\Theta)$ and an increase of overburden pressure always reduces an equal amount of moisture ratio and void ratio $(d e / d P=d \Theta / d P \neq$ 0 for $P \geq 0$ ). But, in the residual shrinkage phase, overburden pressure can be increased without changing the moisture ratio, while changing the void ratio $(\partial \Theta / \partial P)_{e}=0$ and $(\partial e / \partial P)_{\theta} \neq 0$ is only possible in the residual phase where $\Theta<\Theta_{a n}$ ). Therefore $\Theta_{I n}$ must lie within the range $\Theta_{d n}<\Theta_{I n}<\Theta_{a n}$ and on the residual surface.

\section{Equilibrium Moisture Profiles in Swelling Soils}

Using the similarity between $d z / d \Theta$ and $d P / d \Theta$, the behavior of these moisture profiles is most conveniently described on the $e=0$ plane using the properties of the function $M_{n}(\Theta)$ described above, as shown in Figures $4 b, 5 b$, and $6 \mathrm{~b}$. Three different equilibrium conditions are described according to the surface moisture ratio $\Theta_{P 0}$ and surface zero overburden pressure $P_{0}=0$. The same method is applicable for any other surface moisture ratios and surface loads.

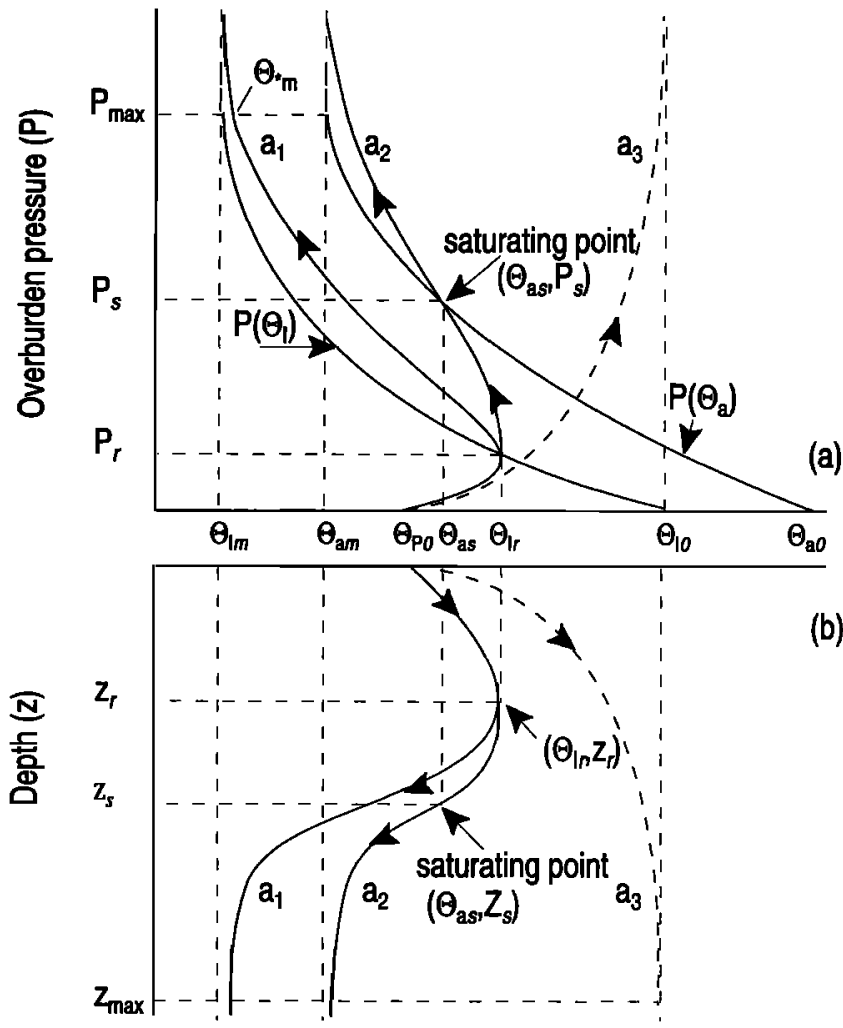

Figure 4. Idealized equilibrium moisture profiles for surface moisture ratio, $\Theta_{P 0}<\Theta_{10}$ and $P_{0}=0$. Curves a3 according to Philip [1969a]. 


\subsection{Case a: When the Surface Moisture Ratio $\Theta_{P 0}$ Lies} in $\Theta_{70}>\Theta_{P 0}>0$ and $P_{0}=0$

This is an unloaded surface where the location $\left(\Theta_{P 0}, P_{0}=0\right)$ is shown on the idealized diagrams of $P$ versus $\Theta$ and $Z$ versus $\Theta$ in Figures 4a and 4b. For $\Theta_{I 0}>\Theta_{P 0}, M_{0}\left(\Theta_{P 0}\right)>0, d z / d \Theta>0$, and $d P / d \Theta>0$ on the ground surface with no load $\left(P_{0}=0\right)$. Therefore $\Theta$ increases with $Z$ and $P$ toward the $P\left(\Theta_{I}\right)$ boundary as long as $P(\Theta)<P\left(\Theta_{I}\right)$. It meets $P\left(\Theta_{I}\right)$, say at $\Theta_{I r}$ as shown in Figure $4 \mathrm{a}$. The point $\Theta_{I r}$ lies on one of the shrinkage curves, say $e_{r}(\Theta)$, which is determined for the overburden pressure $P_{r}$ (equivalent depth $\left.Z_{r}\right)$. At the point $\left(\Theta_{I r}, P_{r}\right), M_{r}\left(\Theta_{I r}\right) \rightarrow+\infty, d P / d \Theta \rightarrow$ $+\infty$, and $d z / d \Theta \rightarrow+\infty$. Therefore the moisture path crossing the $P\left(\Theta_{I}\right)$ boundary will always become parallel to the $P$ and $Z$ axes as shown in Figures $4 \mathrm{a}$ and $4 \mathrm{~b}$. When $P(\Theta)>P\left(\Theta_{I}\right), M(\Theta)=$ $d z / d \Theta \approx d P / d \Theta<0$, and therefore the moisture path continues toward the $P$ axis but away from the $P\left(\Theta_{I}\right)$, because on $P\left(\Theta_{I}\right)$, $d P / d \Theta \rightarrow+\infty$. Further continuation of the moisture path could either intersect the $P\left(\Theta_{a}\right)$ boundary, say at $\Theta_{a s}$ (on the shrinkage curve, $e_{s}(\Theta)$ ), which is determined for $P_{s}$, (equivalent depth $Z_{s}$ ), or cross the shrinkage curve $e_{m}(\Theta)$ for $P=P_{\max }\left(Z_{m}\right)$ at a point $\Theta_{*_{m}}$ in the range $\Theta_{a m}>\Theta_{*_{m}}>\Theta_{I m}$ as shown by a1 in Figure 4a. The moisture path will not cross the $P\left(\Theta_{I}\right)$ boundary for $P>P_{r}$ because on $P\left(\Theta_{I}\right), d P / d \Theta \rightarrow+\infty$ and $d z / d \Theta \rightarrow+\infty$. On the other hand, if it intersects $P\left(\Theta_{a}\right)$ at $\Theta_{a s}$, which is on the load surface, the soil becomes saturated. Thereafter the moisture path is forced to remain on the load surface for $P \geq P_{s}$, because on the load surface, $e=\Theta$, and further increases in $P$ and $Z$ will result in a similar reduction of both $e$ and $\Theta$, forcing the soil to remain saturated and on the load surface with increasing depth. As the moisture gradient is still negative in $P(\Theta)>P\left(\Theta_{I}\right)$, the moisture

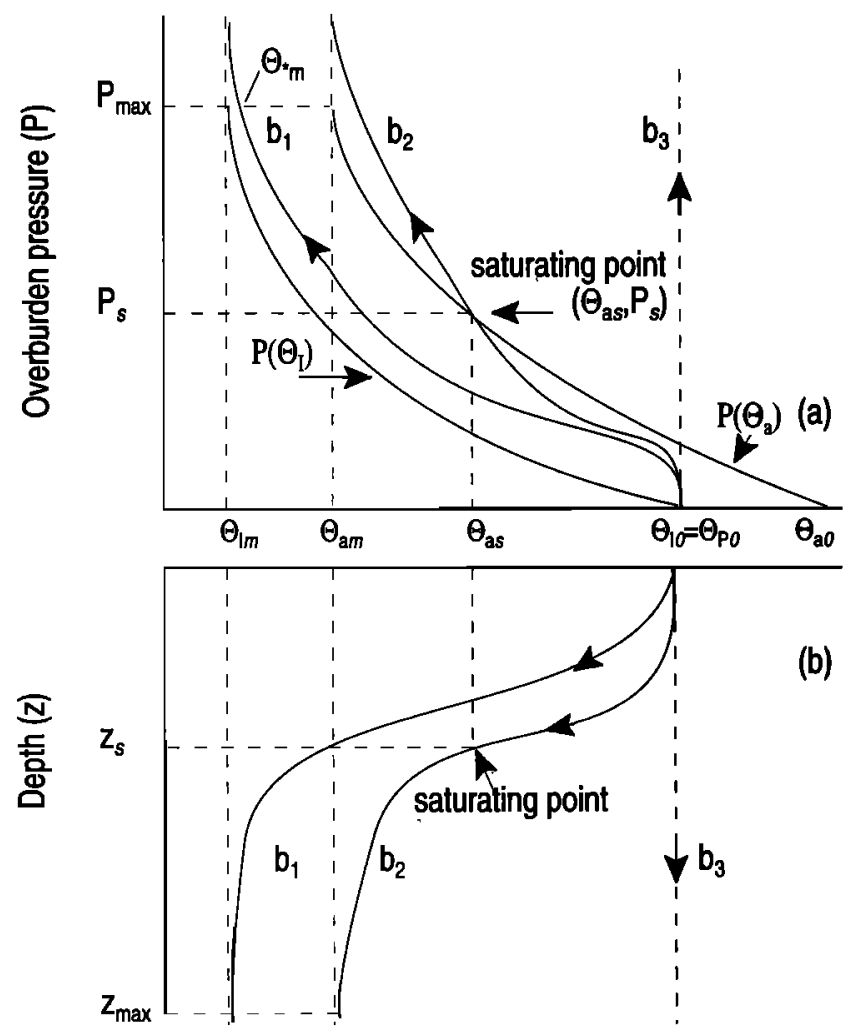

Figure 5. Idealized equilibrium moisture profiles for surface moisture ratio, $\Theta_{P 0}=\Theta_{I 0}$ and $P_{0}=0$. Curves b3 according to Philip [1969a].

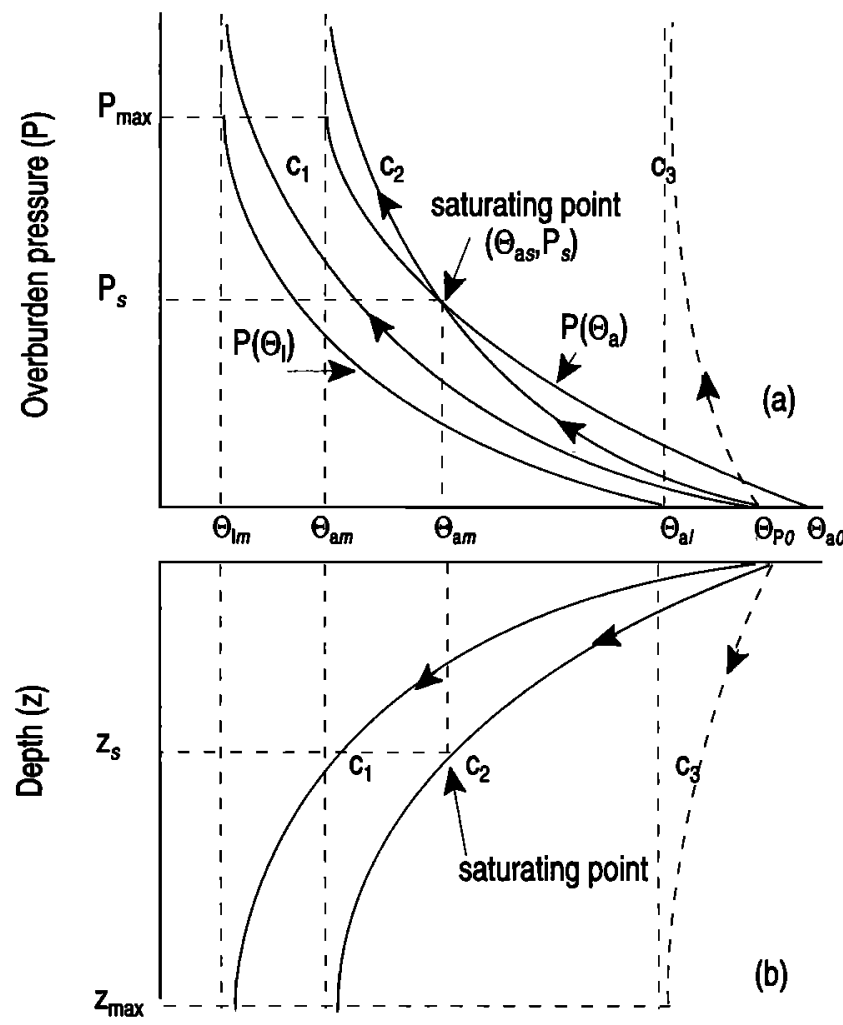

Figure 6. Idealized equilibrium moisture profiles for surface moisture ratio, $\Theta_{P 0}, \Theta_{a 0}>\Theta_{P 0}>\Theta_{I 0}$ and $P_{0}=0$. Curves c3 according to Philip [1969a].

path approaches the $P\left(\Theta_{a}\right)$ boundary once again, for $P>P_{s}$, where it remains saturated at $\Theta_{a m}$ throughout the depth for $P>$ $P_{\max }$ on the load surface as shown in Figures $4 \mathrm{a}$ and $4 \mathrm{~b}$.

If the path reaches a point $\Theta_{* m}$ where $\Theta_{I m}<\Theta_{* m}<\Theta_{a m}$, as shown by a1 in Figure $4 \mathrm{a}$, due to the negative moisture gradient for $P(\Theta)>P\left(\Theta_{I}\right)$ the moisture path continues toward the $P\left(\Theta_{I}\right)$ boundary, which is parallel to the $P$ axis for $P>P_{\max }$ at $\Theta_{I m}$, where it remains unsaturated throughout the entire depth. Philip's [1969a] moisture profiles under similar conditions are shown by $a 3$ in Figures $4 \mathrm{a}$ and $4 \mathrm{~b}$. If a surface load $P_{l}$ is present on the surface, the moisture path begins at a point $P_{0}=P_{l}$ and $\Theta=\Theta_{0 l}$.

\subsection{Case b: $\Theta_{P 0}=\Theta_{r 0}$, and $P_{0}=0$}

This point is located on the $\Theta$ axis as shown in Figures 5a and $5 \mathrm{~b}$. At $\Theta_{I 0}$ where the boundary $P\left(\Theta_{I}\right)$ begins, $M_{0}\left(\Theta_{I 0}\right)=$ $d z / d \Theta= \pm \infty$. Soon after the moisture path begins parallel to the $P$ axis at $\Theta_{I 0}$, it enters the region $P\left(\Theta_{I}\right)<P(\Theta)<$ $P\left(\Theta_{a}\right)$ where the moisture gradient becomes negative. Thereafter, the behavior is identical to that in case a above. As $\Theta$ decreases with depth, it reaches either the $P\left(\Theta_{a}\right)$ boundary in $P<P_{\max }$ or the $P\left(\Theta_{I}\right)$ boundary in $P>P_{\text {max }}$. If it intersects $P\left(\Theta_{a}\right)$ in $P<P_{\max }$ at $\Theta_{a s}$ where it becomes saturated, it will remain saturated on the load surface throughout the depth until it reaches $\Theta_{a m}$ once again for $P>P_{s}$ (path b2), where it remains saturated. If it does not intersect $P\left(\Theta_{a}\right)$ for $P<$ $P_{\text {max }}$, it will be unsaturated throughout the entire depth and will reach $\Theta_{I m}$ for $P>P_{r}$, where it remains unsaturated (path b1). The equilibrium moisture profiles of Philip [1969a] under similar conditions are shown by b3 in Figures $5 \mathrm{a}$ and $5 \mathrm{~b}$. 


\subsection{Case c: $\Theta_{a 0}>\Theta_{P 0}>\Theta_{10}$ and $P_{0}=0$}

In this case the moisture gradient is negative from the beginning at $P_{0}=0$. Therefore the moisture path reaches $P\left(\Theta_{a}\right)$ at $\Theta_{a s}$, where it becomes saturated and will remain saturated throughout the entire depth (Figure 6) and reach $P\left(\Theta_{a}\right)$ once again at depths $P>P_{s}$ (path c2). The moisture ratio at depths where $P \geq P_{\max }$ may become saturated (c2) or unsaturated (c1) depending on whether the moisture path intersects $P\left(\Theta_{a}\right)$ or not, as in cases a and $b$. If the surface soil is saturated where $\Theta_{P 0}>\Theta_{a 0}$, the entire moisture path lies on the load surface until it reaches $\Theta_{a m}$ for $P>P_{s}$, where the soil remains saturated.

Equilibrium moisture profiles for any other equilibrium conditions can be traced using the same pracedure and the given surface conditions.

\section{Conclusion}

The equilibrium moisture profiles in swelling soil have been shown to be different from those predicted by earlier authors due to the effects of overburden pressure on the volume change. Soil at great depths may be either saturated or unsaturated. It is clear that if the soil becomes saturated, it will remain saturated at $\Theta_{a m}$ at depths where $P>P_{\max }$ as shown by profile a2 in Figures $4 a$ and $4 b$. However, soil at great depths could be unsaturated and remain unsaturated at a moisture ratio $\Theta_{I m}$ for large depths $\left(P>P_{\max }\right)$ as shown by profile a1 in Figures $4 \mathrm{a}$ and $4 \mathrm{~b}$. However, only two equilibrium moisture ratios exist at great depth in swelling soils. Those two moisture ratios, $\Theta_{I m}$ and $\Theta_{a m}$, are very small, as they are determined for $\boldsymbol{P}_{\max }$. Moisture profiles under equilibrium conditions can be determined for a given surface moisture ratio and the loading, if the details of the shrinkage surface are known. The shrinkage surface can be determined from a series of shrinkage curves under one-dimensional volume change obtained for different overburden pressures from $P=0$ to the overburden pressure relevant to the required depth. Once the equilibrium moisture profile is determined for a soil with a surface load (e.g., a civil engineering structure), an $e(\Theta, P)$ relationship for the entire depth is known. This relationship can then be used to evaluate the void ratio change along the entire depth (i.e., the total settlement) at gravitational moisture equilibrium.

\section{References}

Chang, R. K., and B. P. Warkentin, Volume change of compacted clay soil aggregates, Soil Sci., 105(2), 106-111, 1968.

Ekanayake, J. C., Soil water movement through swelling soils, Ph.D. thesis, Lincoln Univ., Canterbury, New Zealand, 1991.

Fox, W. E., A study of bulk density and water in a swelling soil, Soil Sci., 98(1), 307-316, 1964.
Giraldez, J. V., and G. Sposito, A general soil volume change equation, II, Effect of load pressure, Soil Sci. Soc. Am. J., 47, 422-425, 1983.

Giraldez, J. V., G. Sposito, and C. Delgado, A general soil volume change equation, I, The two-parameter model, Soil Sci. Soc. Am. J., 47, 419-422, 1983.

Groenevelt, P. H., and G. H. Bolt, Water retention in soil, Soil Sci., 113(4), 238-245, 1972.

Haines, W. B., The volume change associated with variations of water content in soil, J. Agric. Res., 13, 296-310, 1923.

Holmes, J. W., Water sorption and swelling of clay blocks, J. Soil Sci., 6(2), 200-207, 1955.

Lauritzen, C. W., and A. J. Stewart, Soil volume changes and accompanying moisture and pore-space relationships, Soil Sci. Soc. Am. Proc., 6, 113-116, 1941.

Mesri, G., and R. E. Olson, Consolidation characteristics of montmorillonite, Geotechnique, 21(4), 341-352, 1971.

Philip, J. R., Moisture equilibrium in the vertical in swelling soils, 1, Basic theory, Aust. J. Soil Res., 7, 99-120, 1969a.

Philip, J. R., Moisture equilibrium in the vertical in swelling soils, II, Applications, Aust. J. Soil Res., 7, 121-141, 1969b.

Philip, J. R., Hydrostatics and hydrodynamics in swelling soils, Water Resour. Res., 5(5), 1070-1077, 1969c.

Philip, J. R., Reply, Water Resour. Res., 6(4), 1248-1251, 1970.

Philip, J. R., Hydrology of swelling soils, in Salinity and Water Use, Proceeding Symposium Australia, edited by T. Talsma and J. R Philip, pp. 125-139, Macmillan, New York, 1971.

Philip, J. R., Recent progress in the theory of irrigation and drainage of swelling soils, paper presented at the 8th Congress of the International Commission on Irrigation and Drainage, Int. Comm. on Irrig. and Drain., Varna, Bulgaria, 1972.

Philip, J. R., and D. E. Smiles, Kinetics of sorption and volume change in three component systems, Aust. J. Soil Res., 7, 1-19, 1969.

Sposito, G., Volume change in swelling clays, Soil Sci., 115(4), 315-320, 1973.

Talsma, T., Moisture profiles in swelling soils, Aust. J. Soil Res., 12, 71-75, 1974

Talsma, T., A note on shrinkage behaviour of a clay paste under various loads, Aust. J. Soil Res., 15, 275-277, 1977.

Tempany, H. A., The shrinkage of soils, J. Agric. Res., 8, 312-333, 1917.

Towner, G. D., The correction of in situ tensiometer readings for overburden pressures in swelling soils, $J$. Soil Sci., 32(4), 499-504, 1981.

Warkentin, B. P., and M. Bozozuk, Shrinking and swelling properties of two Canadian clays, Proc. Int. Conf. Soil Mech. Found. Eng., 1, 5, 851-855, 1961.

Yong, R. N., and B. P. Warkentin, Soil Properties and Behaviour, Elsevier Sci., New York, 1975.

Youngs, E. G., and G. D. Towner, Comments on "Hydrostatics and hydrodynamics in swelling soils" by J. R. Philip, Water Resourc. Res., 6(4), 1246-1247, 1970.

J. C. Ekanayake, Manaaki Whenua-Landcare Research New Zealand Ltd., P. O. Box 69, Canterbury Agriculture and Science Centre, Gerald Street, Lincoln, New Zealand. (e-mail: ekanayaj@landcare.cri.nz) D. J. Painter, Department of Natural Resources Engineering, P. O. Box 84, Lincoln University, Canterbury, New Zealand. (e-mail:painter@ lincoln.ac.nz)

(Received May 9, 1995; accepted May 24, 1995.) 